\title{
Estimación y análisis de la demanda hídrica de la subcuenca del Río Estibaná como parte del balance hídrico (2018-2019)
}

\author{
Martínez, Arcenio \\ Facultad de Ingeniería Civil, Universidad Tecnológica de Panamá \\ Panamá, Panamá \\ arcenio.martz@gmail.com \\ Rodríguez, Zuri \\ Rectoría, Universidad Tecnológica de Panamá \\ Panamá, Panamá \\ zuri.rodriguez@utp.ac.pa \\ Fábrega Duque, José \\ Centro de Investigaciones Hidráulicas e Hidrotécnicas, Universidad Tecnológica de Panamá \\ Miembro del Sistema Nacional de Investigación de Panamá (SNI) \\ Panamá, Panamá \\ https://orcid.org/0000-0003-1536-0386
}

\begin{abstract}
The Estibana sub-catchment has an area of $296 \mathrm{~km}^{2}$ and is located within the basin of the La Villa River, province of Los Santos, in the Dry Arc. It is the region with the least water availability in the country, with an average rainfall of $1400 \mathrm{~mm} /$ year. Its population depends mainly on agriculture and livestock. Water is currently supplied to the population through rural aqueducts, water treatment plants and wells. The problem lies mainly in dry seasons, especially in the years that El Niño phenomenon occurs, causing extensive droughts. Therefore, the objective of this work was to determine the water demand in three sectors (population, livestock and agriculture) for the period of April 2018 and March 2019 (hydrological year). The estimated population in 2020 was 9285. In 2018, 32000 cattle and 890 hectares of crop field were registered. Livestock demand was calculated based on growth, age and temperature, where per capita consumption was estimated for three scenarios, where the mean value was 79 L/bov-day. Agricultural demand took as a reference the potential evapotranspiration, crop coefficients and irrigation efficiency, crop area and periods of growth. A total demand of $6.32 \mathrm{Mm} 3 /$ year was obtained where the
\end{abstract}


highest consumption occurs in agriculture with $81 \%$. However, the water demand in the critical months is $1.58 \mathrm{Mm} 3$, where the flow of the river decreases and the wells and water treatment plants decrease the supply capacity, so it is necessary an efficient management of the water resource.

Keywords: Water Demand, Dry Arc, Agriculture, Livestock, Drought

\section{Resumen}

La subcuenca del río Estibaná tiene una superficie $296 \mathrm{~km}^{2}$ y está ubicada dentro de la cuenca del río La Villa, provincia de Los Santos, en el Arco Seco, región con menos disponibilidad hídrica del país, con precipitaciones promedio de 1400 mm/año. Su población depende de la agricultura y ganadería principalmente. Actualmente se suministra agua a la población mediante acueductos rurales, plantas potabilizadoras y pozos. El problema radica en las épocas secas, especialmente, en los años que se da el fenómeno de El Niño, provocando sequías extensas. Por lo tanto, el objetivo de este trabajo fue determinar la demanda hídrica en tres sectores (población, ganadería y agricultura) para el período de abril 2018 y marzo 2019 (año hidrológico). La población estimada a 2020 fue de 9285 habitantes. En 2018 se registraron 32000 bovinos y 890 hectáreas de cultivo. La demanda ganadera fue calculada en base al crecimiento, edad y temperatura, donde se estimó un consumo per cápita para tres escenarios; donde el valor promedio fue de 79 L/bov-día. La demanda agrícola tomó de referencia la evapotranspiración potencial, coeficientes de cultivo y eficiencia de riego, superficie sembrada y períodos de crecimiento. Se obtuvo una demanda total de 6.32 $\mathrm{Mm3} / \mathrm{año}$ donde el mayor consumo se da en la agricultura con un $81 \%$. No obstante, la demanda hídrica en los meses críticos es de $1.58 \mathrm{M}$, donde el caudal del río disminuye y los pozos y plantas potabilizadoras disminuyen la capacidad de abastecimiento, por lo que es necesaria una gestión eficiente del recurso hídrico.

Palabras claves: Demanda Hídrica, Arco Seco, Agricultura, Ganadería, Sequía.

\section{INTRODUCCIÓN}

En años recientes, debido a la falta de adecuaciones de infraestructura que garanticen el suministro de agua y permitan una mejor adaptación al cambio climático [1], la agricultura y la ganadería de esta región han sido afectadas principalmente en la época seca. Las pocas precipitaciones que se registran y las sequías prolongadas, sobre todo en los años de El 
Niño, el cual se manifiesta de manera cíclica de 2 a 7 años [2] produciendo disminuciones considerables en los caudales del río Estibaná y retrasando las primeras lluvias. Una de las alternativas desarrolladas en la región es habilitar pozos a lo largo de la subcuenca que suministren agua a las comunidades rurales de la zona. Sin embargo, la construcción de éstos ha sido desordenada, derivando en una sobreexplotación en la región [3].

Para estimar la magnitud de la demanda de agua dentro de la subcuenca del río Estibaná, se estudió el comportamiento social, económico y cultural de la zona. En esta región, se realizó un análisis de la demanda doméstica que es suministrada diariamente en la región. También fueron evaluadas las distintas actividades económicas de importancia tales como: la agricultura y la ganadería

\section{METOdOLOGÍA}

\section{A. Demanda doméstica}

Para obtener un valor aproximado de cuánto es suministrado por las infraestructuras y sistemas de abastecimiento de agua potable, se procesaron datos del Instituto Nacional de Estadística y Censo (INEC) para la estimación de la demanda poblacional. Dado que, el último censo poblacional fue realizado en el año 2010, se trabajó con las estimaciones futuras de crecimiento hasta el año 2020. Para producir un valor aproximado de cuánto consume la población al año, se empleó un consumo per cápita (CPC) de 30 gal/Hab-día, correspondiente a zonas rurales [4].

$$
\mathrm{Dp}=\mathrm{CPC} * \mathrm{Po} * \mathrm{t}
$$

\section{B. Demanda agrícola}

Con la ayuda de bases de datos de tres agencias del MIDA (Macaracas, Tres Quebradas y La Villa), se seleccionaron los cultivos principales para el período 2018-2019. Los coeficientes de cultivo (Kc) se obtuvieron del Manual de Evapotranspiración de la FAO [5]"type":"book"\},"uris":[“http://www.mendeley. com/documents/?uuid=cf592d68-5790-49e7-9736-dcbf466d97e2"]\}],"mendeley ":\{“formattedCitation":"[5]"," plainTextFormattedCitation":"[5]","previouslyFormattedCitation":"[5]"\},"properties":\{“noteIndex":0\},"schema":"https://github.com/citationstyle-language/schema/raw/master/csl-citation.json"\}. La ETP se obtuvo de los datos de un balance hídrico en tiempo real de la página de ETESA [6]. La eficiencia del riego se obtuvo de documentos técnicos [7].

$$
D a_{\text {Mensual }}=\frac{K c \times E T P}{K r} \times \frac{A_{C}}{A_{T}}
$$




\section{Demanda ganadera}

Según datos del Censo Nacional Agropecuario 2017, existen 34610 cabezas de ganado. Para saber el consumo de agua de cada animal, se realizó una cuantificación en función del crecimiento, el tiempo y la temperatura [8]. Debido a que el crecimiento puede ser distinto en cada etapa de la vida del animal, se estudiaron tres comportamientos. Primero, animales recién nacidos hasta alcanzar un año de vida. Segundo, animales entre 1 y 2 años. Finalmente, se consideraron los animales adultos mayores de dos años. Se estimó el volumen de agua consumido mediante la elaboración de gráficos de consumo diario versus tiempo.

$$
\begin{gathered}
D g=\sum\left(C p_{i} * B o v\right) \\
C p_{i}=\int_{t i}^{t f} f(x)
\end{gathered}
$$

\section{RESULTADOS}

Los resultados de la demanda fueron calibrados mediante la aplicación de una encuesta que fue realizada en distintas giras y épocas para conocer el comportamiento y distribución de la demanda lo largo de la subcuenca del Río Estibaná.

Para la estimación poblacional, el INEC considera los datos históricos y tasas de crecimiento demográfico. Conociendo la población estimada de 9285 , se obtiene un consumo poblacional aproximado de 380797 m3/año.

La tabla 1 presenta una demanda anual aproximada de los cultivos de $17.51 \mathrm{~mm}$, lo cual equivale a 5144967.42 /año. Dentro de esta demanda se incluyen tanto los cultivos regados por lluvia como los que se riegan por goteo, el cual fue el método de riego más usado por los agricultores, sobre todo en época seca para el riego de cucurbitáceas y solanáceas. De las encuestas se obtuvo que el riego por goteo es el más empleado entre diciembre - marzo (época seca). Teóricamente el riego por goteo es el más recomendado, ya que posee una eficiencia del $90 \%$ (coeficiente de riego $\mathrm{Kr}=0.90$ )(Leonardo, 2015). A pesar de que el maíz es el cultivo más común en esta subcuenca, la cantidad de hectáreas ha disminuido con respecto al 2012 (1510.85 ha vs $835.50 \mathrm{ha}$ ), e incluso varios agricultores de otros cultivos han abandonado esta actividad, ya que se han visto duramente afectados por las sequías. 
Tabla 1. Distribución de la demanda (Da) en los cultivos principales de la subcuenca del río Estibaná.

\begin{tabular}{c|ccc}
\hline Cultivos & A (Ha) & ${ }^{*}$ Da () & ${ }^{*}$ Da $(\mathbf{m m} /$ día $)$ \\
\hline Tomate & 3.00 & 12452.00 & 0.04 \\
Ají & 6.34 & 28912.78 & 0.10 \\
Pepino & 2.50 & 10470.25 & 0.04 \\
Melón & 8.25 & 31311.22 & 0.11 \\
Zapallo & 9.93 & 48168.23 & 0.14 \\
Sandía & 15.43 & 39040.98 & 0.13 \\
Maíz & 835.50 & 4915156.89 & 16.76 \\
Arroz & 9.00 & 59455.06 & 0.21 \\
Total & $\mathbf{8 9 0 . 0 0}$ & $\mathbf{5 1 4 4} \mathbf{9 6 7 . 4 2}$ & $\mathbf{1 7 . 5 1}$ \\
\hline
\end{tabular}

La relación entre el crecimiento progresivo de los bovinos menores a un año y a una temperatura promedio de $30^{\circ} \mathrm{C}$, arrojó un CPC de $45 \mathrm{~L} /$ bov-día (gráfico 1(a)). Los bovinos que poseen más de un año de vida y menos de 2 años, presentaron un CPC equivalente a $68 \mathrm{~L} /$ bovino-día (gráfico 1(b)), mientras que los bovinos que superan un periodo de vida mayor a 3 años pueden llegar a alcanzar unos 128 L / bovino-día (gráfico 1(c)).

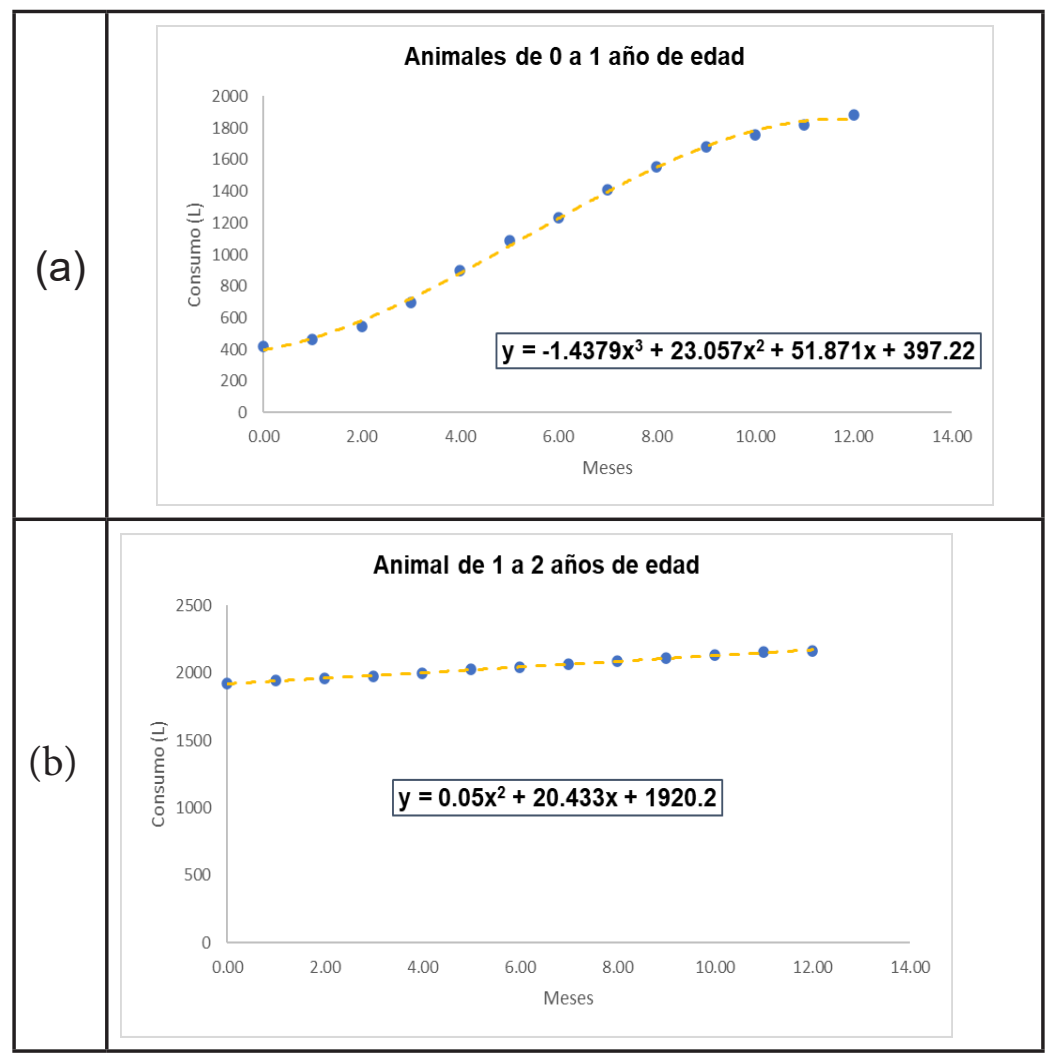




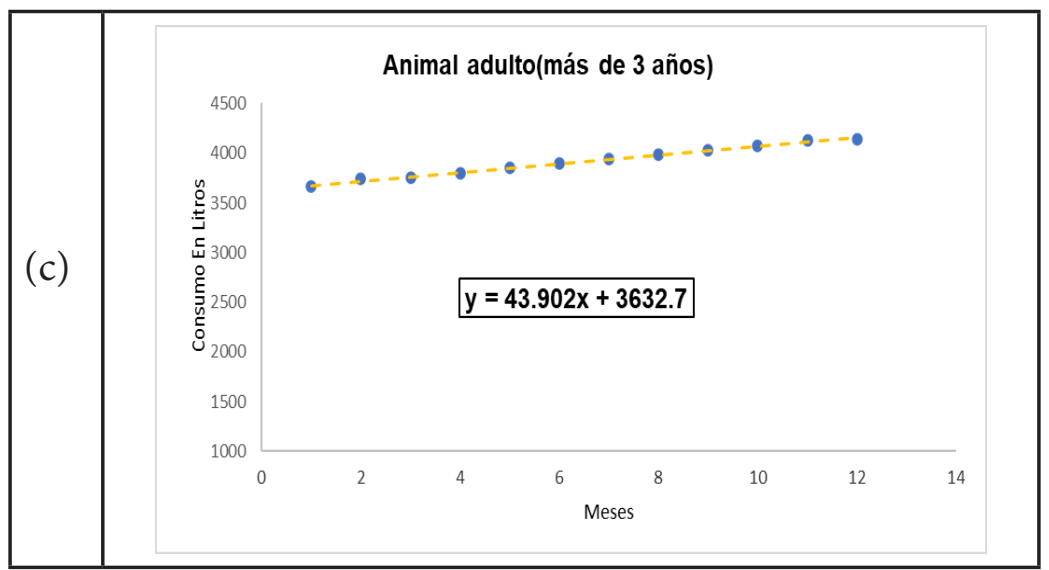

Gráfica 1. Consumo de animales bovinos en los tres comportamientos analizados: (a) 0-1 año, (b) 1-2 años y (c) más de 2 años.

En la gráfica 2 se observa que la mayor demanda corresponde a la agrícola con un $81 \%$ y luego la ganadera con un $13 \%$. La demanda doméstica resultó en un $6 \%$ de la demanda total, sin embargo, continúa siendo la variable más delicada por tratarse directamente de la población, ya que afectaciones en la disponibilidad hídrica de la subcuenca limita sus necesidades básicas y de realizar sus actividades de subsistencia.

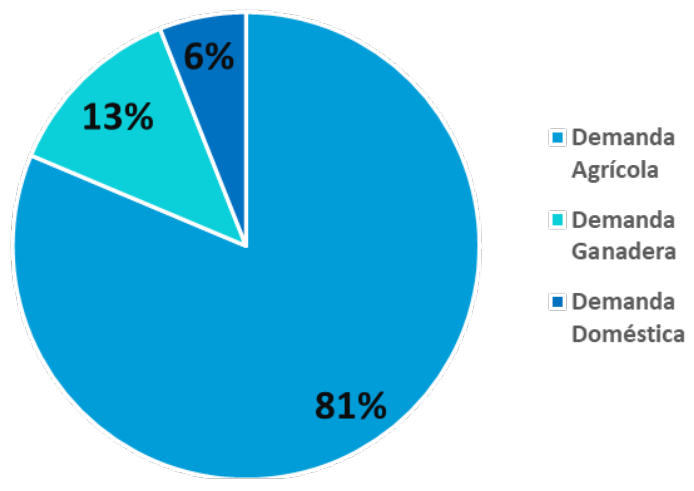

Gráfica 2. Distribución anual de la Demanda Hídrica para el período 2018-2019 en la subcuenca del río Estibaná.

\section{CONCLUSIONES}

A pesar de que la demanda de consumo doméstico en la zona de estudio es pequeña en comparación con la ganadera y agrícola, esta se ve extremadamente afectada durante los meses de escases lluviosa, ya que, al disminuir el caudal del río, las infraestructuras existentes tienden a trabajar a capacidad mínima. La mayor demanda viene de las actividades agrícolas, y se ve principalmente afectada durante los primeros meses del año cuando se 
dan pocas precipitaciones; por ende, los niveles de los afluentes disminuyen, siendo el único suministro los pozos profundos. En cuanto a la demanda de ganado bovino se obtuvo un consumo promedio alrededor de 79 L/bovino-día. Es necesario ampliar la capacidad de almacenamiento del agua mediante nuevas políticas e infraestructuras, para asegurar una gestión sostenible del recurso hídrico en la subcuenca.

\section{Referencias}

[1] MiAmbiente, "Tercera Comunicación Nacional sobre Cambio Climático en Panamá," 2018.

[2] H. ETESA, "El Fenómeno de El Niño," Panamá, 2020.

[3] J. Espinoza and F. Tabora, "Situación de los Recursos Hídricos en Centroamérica- Panamá," Tegucigalpa, Honduras, 2015.

[4] ANAM, "Cuenca Hidrográfica 128, Río La Villa, Evaluación de las Componentes del Balance Hídrico," 2008.

[5] R. G. Allen, L. S. Pereira, D. Raes, and M. Smith, Evapotranspiración del cultivo. Guía para la determinación del requerimiento de agua del cultivo, no. January. 2006.

[6] ETESA, "HIDROMET.COM," 2019. .

[7] I. A. N. Leonardo, “Manual del Cálculo de Eficiencia para Sistemas de Riego," Lima, 2015.

[8] E. Duarte, "Uso del Agua en establecimientos agropecuarios. Planificación del sistema de abrevadero," Revista Plan Agropecuario, vol. 140, no. Parte II, pp. 38-43, 2011.

\section{Autorización y Licencia CC}

Los autores autorizan a APANAC XVIII a publicar el artículo en las actas de la conferencia en Acceso Abierto (Open Access) en diversos formatos digitales (PDF, HTML, EPUB) e integrarlos en diversas plataformas online como repositorios y bases de datos bajo la licencia CC:

Attribution-NonCommercial-ShareAlike 4.0 International (CC BY-NC-SA 4.0) https://creativecommons. org/licenses/by-nc-sa/4.0/.

Ni APANAC XVIII ni los editores son responsables ni del contenido ni de las implicaciones de lo expresado en el artículo. 